\title{
Rendimiento de las pruebas diagnósticas en el sangrado del intestino delgado
}

\section{Performance of diagnostic tests on bleeding from the small intestine}

Raúl Antonio Cañadas-Garrido, MD. ${ }^{1}$

Médico internista, gastroenterólogo. Profesor Asociado de Medicina, Pontificia Universidad Javeriana. Gastroenterólogo, Hospital Universitario San Ignacio. Jefe de la Unidad de Endoscopia digestiva, Clínica de Marly. Expresidente de la Asociación Colombiana de Endoscopia Digestiva. Bogotá D. C., Colombia.

*Correspondencia:

Raúl Antonio Cañadas-Garrido, MD

raulcanadas@gmail.com

Fecha recibido: $27 / 05 / 20$

Fecha aceptado: 08/06/20
El intestino delgado históricamente ha sido considerado un órgano de difícil acceso, por lo que durante muchos años fue llamado la caja de pandora del tracto gastrointestinal; pero la historia cambió, y en la actualidad se pueden identificar lesiones que previamente eran inalcanzables por métodos endoscópicos. Posiblemente, el gran paso en el desarrollo estuvo marcado por la llegada de la videocápsula endoscópica (VCE), que desde su introducción en 2001 revolucionó la endoscopia del intestino delgado y, desde entonces, gracias a su perfeccionamiento, se lograron tasas increíbles para la detección de lesiones que le permitieron consolidarse en lo que es actualmente: la principal herramienta diagnóstica en lesiones del intestino delgado basado en el objetivo fundamental de lograr el estudio completo de los segmentos intestinales, alcanzando hasta el momento cifras de evaluación completa del intestino delgado en $70 \%$ a $90 \%$ de los pacientes y soportado en una baja tasa de error diagnóstico, que la promueve como un excelente método diagnóstico.

Es tan importante la evolución de la VCE en el desarrollo de las imágenes que abrió esa caja de pandora y se empezó a hablar de diagnósticos endoscópicos nunca antes vistos de manera directa; y fue tal su impacto que, dentro de su principal indicación, el estudio de sangrado gastrointestinal de origen oscuro, hizo que el enfoque diagnóstico también cambiara, pues muchos de los pacientes con sangrado de etiología incierta dejaron de serlo en un porcentaje tal que claramente incentivó un cambio en la terminología. En el fondo, habría cada vez menos casos considerados como de origen oscuro y se avanzaría más al desarrollo de modalidades de tratamiento intervencionista para todos estos nuevos hallazgos que se tendrían que afrontar siendo consecuentes con su desarrollo.

Entonces, la definición de sangrado gastrointestinal cambió y se creó un término nuevo: el sangrado del intestino delgado, definido como el sangrado originado más allá de la ampolla de Vater hasta la válvula ileocecal, que desplazó en parte a la definición previa de sangrado digestivo bajo, que incluía todos los trastornos originados desde el ligamento de Treitz hasta el ano. Pero aún así seguirán existiendo casos de sangrado oscuro, posiblemente debido a que el intestino delgado tiene en promedio de 6 a 7 metros de longitud y 2,5 cm de diámetro, lo que predispone a estudios incompletos aun con la mejor tecnología, así como también se prevén los falsos negativos de la prueba junto con el misterio de la enfermedad en el ser humano.

De acuerdo con los avances en el estudio del intestino delgado, el término sangrado de origen oscuro actualmente queda reservado solo para aquellos pacientes que a pesar de tener exámenes endoscópicos negativos (endoscopia alta y colonoscopia), tienen estu- 
dios complementarios de intestino delgado (VCE, enteroscopia o imágenes radiológicas) que no evidencian la causa de sangrado (1). En ese sentido, esta evolución indudablemente cambiará la estadística previa, pues la tendencia de nuevas definiciones existe solo desde hace algunos años, por lo que la mayoría de los estudios de rendimiento está soportada en las previas definiciones de sangrado gastrointestinal de origen oscuro, manifiesto y oculto, que muy pocas publicaciones en los últimos años han utilizado.

Conjuntamente y casi de manera simultánea llega la revolución de la enteroscopia asistida con dispositivos como una herramienta fundamental en el estudio y tratamiento de patologías del intestino delgado, lo que complementa la imposibilidad de intervención de la VCE. Entonces, la enteroscopia surge no como una competencia sino más bien como una necesidad de cumplir con éxito todos los logros en la evolución de las imágenes. Se puede decir que la enteroscopia asistida aparece como una tecnología hermana, complementaria, con pros y contras relevantes, y su capacidad de intervencionismo; pero también con su principal desventaja: una técnica marcadamente invasiva, pero que en conjunto con la VCE ha permitido que la percepción incrédula sobre las enfermedades del intestino delgado actualmente se dirija al acercamiento y comprensión de estas patologías. En ese sentido se puede proponer una reflexión: la VCE no sería tan apreciada sino existiera la enteroscopia asistida y, de hecho, la enteroscopia no sería tan reconocida sin la VCE (debido a que utiliza una sola vía de exploración y su alto valor predictivo negativo [VPN]), que permite evitar enteroscopias invasivas en pacientes con una baja probabilidad de hallazgos positivos en el intestino delgado previa al examen. En conclusión, son simbióticas, dependen la una de la otra y, si se traduce la agudeza diagnóstica en números, el rendimiento diagnóstico de la VCE y la enteroscopia es similar en su principal indicación: el sangrado manifiesto, que alcanza cifras del $92 \%$. Se podría asegurar que no compiten, son igualmente buenas, pero en algunas situaciones especiales puede haber una inclinación a favor de alguna de ellas.

Para evaluar números y objetivar resultados, es necesario revisar unos años atrás para estudiar el rendimiento diagnóstico que cada una de las pruebas ha mostrado con su desarrollo y globalización en las unidades de endoscopia. Desde que estas pruebas han estado disponibles, los endoscopistas han tenido muchas experiencias positivas con su manejo, debido a que han podido encontrar muchas imágenes que anteriormente eran imposibles de obtener.

Las aplicaciones actuales de la cápsula endoscópica han ido creciendo desde su introducción y se han conservado las ventajas iniciales, que incluyen la adherencia por su naturaleza menos invasiva, el hecho de no requerir anestesia ni sedación, la capacidad de estudio completo del intestino delgado y el excelente perfil de seguridad. Por estas razones se recomienda la VCE del intestino delgado como la investigación de primera línea en pacientes con sangrado gastrointestinal de origen oscuro (recomendación fuerte, calidad de evidencia moderada) (2).

Gracias a esta oportunidad de escribir el editorial, antes de entrar a la revisión bibliográfica aprovecho para expresar que, independientemente de las ventajas de todas las pruebas para el estudio del sangrado del intestino delgado que se mencionan más adelante, ninguna va a reemplazar el juicio clínico acompañado de la adecuada selección de los pacientes, esto impactará directamente en las tasas de rendimiento diagnóstico y será clave en la percepción de la etiología que se está explorando.

En el estudio de patologías del intestino delgado es importante pensar en orden sobre la epidemiología y la posibilidad de la aparición de lesiones de acuerdo con la edad del paciente, mas no de sexo ni etnia. La edad es clave en la determinación de probables etiologías del sangrado del intestino delgado. Cuando se introducen todas las etiologías en grupos, las causas vasculares serán las más frecuentes, seguidas de las causas inflamatorias y neoplásicas; pero al discriminar por edades las cosas cambian radicalmente. En pacientes menores de 40 años, las causas más importantes serán las inflamatorias (enfermedad de Crohn, enteritis infecciosa, úlceras intestinales y divertículo de Meckel, y son menos probables las lesiones tumorales y las vasculares como la angiectasia). En mayores de 40 años, las causas más probables son la angiectasia, seguida por las causas inflamatorias (úlceras por antiinflamatorios no esteroideos [AINE] y enteritis inespecíficas) y las neoplasias, entre ellas el adenocarcinoma (el más frecuente de localización más proximal en el duodeno y yeyuno proximal), el linfoma, el tumor carcinoide y el sarcoma, estos 3 últimos de localización distal en yeyuno-íleon.

Adicionalmente, en el enfoque clínico es necesario explorar los factores de riesgo para el sangrado, entre ellos alteraciones de la coagulación como en la enfermedad de Von Willebrand y enfermedad cardíaca valvular aórtica, por su asociación con lesiones vasculares, antecedentes de insuficiencia renal crónica (IRC) en hemodiálisis, hipertensión portal, uso de medicamentos: ácido acetilsalicílico (ASA), los AINE, anticoagulantes, antiplaquetarios, sospecha clínica de vasculitis y amiloidosis; y procedimientos previos: biopsia hepática, trasplante hepático, reparación de aneurisma abdominal, resección intestinal y radioterapia; sin dejar atrás los antecedentes familiares de enfermedad inflamatoria, poliposis y telangiectasia hereditaria familiar. Todo esto, aunque sea reiterativo, es la clave para mejorar el rendimiento diagnóstico de las pruebas del intestino delgado al momento de seleccionar los pacientes.

También los invito a abrir la mente para pensar en que existen patologías infrecuentes que se están reconociendo 
en imágenes más repetidamente, entre ellas la enfermedad de Rendu-Osler-Weber, síndrome de nevo azul, sarcoma de Kaposi, enteropatía portal, poliposis hereditaria, hemobilia, Dieulafoy, entre otras.

La etiología vascular es la causa global más frecuente de sangrado digestivo de intestino medio, por lo que la angiectasia merece una mención especial, que constituye la malformación vascular más común del tracto gastrointestinal y la causa más frecuente de sangrado del intestino delgado. Las altas tasas de resangrado son un gran problema (17 $\%-40 \%$ ), sin que se determine cuáles son los predictores de resangrado, excepto al parecer por la presencia de lesiones múltiples definidas como mayores a 3 , y por ello es preciso mencionarlas como parte de la problemática. Es fundamental que aquellos que interpretan las VCE se esfuercen al máximo en la adecuada identificación de estas lesiones, idealmente de acuerdo con la clasificación de Saurin, especificando qué tipo de lesión vascular observa, clasificarla y, en caso de angiodisplasia típica, señalarla como una lesión con alto potencial de sangrado y diferenciarlas semiológicamente de las manchas rojas con potencial incierto para sangrado. De este modo, posiblemente se eviten procedimientos invasivos innecesarios y que repercutan en el rendimiento de las pruebas o se subestimen las verdaderas angiectasias indicativas de terapia.

En cuanto al plano de evidencia relacionado con el rendimiento de las pruebas en el sangrado del intestino delgado, se debe entender que el patrón de oro es la enteroscopia intraoperatoria, que tiene tasas importantes de morbimortalidad y, por esta razón, los parámetros para evaluar el rendimiento de la VCE son indirectos al no tener ese patrón estándar de comparación (3).

Varias guías nacionales e internacionales recomiendan la VCE como el primer estudio diagnóstico que debe realizarse en caso de sangrado del intestino delgado, enfatizando en que debe llevarse a cabo en los primeros 14 días de inicio del cuadro o tan pronto como sea posible después del episodio de sangrado para maximizar su rendimiento (4). En este período se identifica la lesión aproximadamente en el 67 $\%$ de casos, mientras que su rendimiento baja considerablemente a un $33 \%$ cuando se realiza a las 3-4 semanas. Podría cambiar la estrategia de manejo entre el $33 \%$ y $66 \%$ de los pacientes, reduciendo el número de hospitalizaciones, las investigaciones adicionales y la necesidad de transfusiones, con el agregado fundamental que incrementa el rendimiento diagnóstico $(73 \%-93 \%)$ y terapéutico $(57 \%-73 \%)$ de la enteroscopia, que se atribuye a la determinación de la vía del procedimiento; de este modo se permite mejorar la identificación de las lesiones vasculares $(5,6)$.

Recomiendo la revisión de la posición de la Sociedad Europea de Endoscopia Gastrointestinal (ESGE) en relación con el papel de la VCE y la enteroscopia en las enfer- medades del intestino delgado, publicado en la revista Endoscopy en 2015, con recomendaciones basadas en la evidencia y que a la fecha continúa plenamente vigente por la solidez de la evidencia científica y la practicidad en su aplicación. Esta guía, de obligatoria lectura cuando se tratan pacientes con enfermedades del intestino medio, manifiesta sus recomendaciones en el estudio del sangrado del intestino delgado, las que expondré en los siguientes apartados de acuerdo con mi percepción de cuáles son las sugerencias más relevantes en el tema.

En primer lugar, se debe resaltar que se observa un progreso importante en la tecnología de la VCE. De hecho, para el 2015 los metaanálisis orientados a evaluar el rendimiento global de la cápsula en hemorragia gastrointestinal oscura era de $61,7 \%$ (intervalo de confianza [IC] 95 $\%: 47,3-76,1$ ), que según el Dr. Pennazio (2) alcanza en la actualidad un rendimiento diagnóstico de $92,3 \%$ en sangrado manifiesto, $67 \%$ cuando hay antecedente de sangrado manifiesto previo y $44,2 \%$ en el sangrado oculto. La literatura describe que la VCE presenta mayor capacidad para encontrar lesiones si existen las siguientes condiciones: hemoglobina $<10 \mathrm{~g}$, duración del sangrado $>6$ meses, sangrado manifiesto, realización de la VCE dentro de las 2 semanas del sangrado (con un máximo rendimiento entre las 48 y 72 horas), más de 1 episodio de hemorragia, sexo masculino, edad $<60$ años, paciente hospitalizado y comorbilidad cardíaca y renal. En segundo lugar, otra cualidad que soporta la VCE como primer estudio de elección es el hecho que después de la VCE negativa el riesgo de resangrado es muy bajo, entre el 5,6\% y el $11 \%$, esto destaca su exactitud y respalda su alto VPN (7).

Por estas razones, debido a la tolerabilidad, eficacia y excelente perfil de seguridad de la VCE, y su potencial para la enteroscopia completa, la ESGE recomienda la VCE de intestino delgado como el examen de primera línea en el estudio del sangrado del intestino delgado antes de considerar la enteroscopia asistida por dispositivo (recomendación fuerte, moderada evidencia de calidad). La evidencia muestra que, al comparar el rendimiento diagnóstico de la VCE con la enteroscopia de doble balón (EDB) en sangrado oscuro, el rendimiento del diagnóstico combinado para la VCE fue del $61,7 \%$ (IC $95 \%: 47,3-76,1$ ) y para la EDB fue del 55,5 \% (IC 95\%: 48,9 a 62,1). En los resultados, la VCE demuestra que tiene una mayor tasa de enteroscopia completa, una tasa más baja de complicaciones, es menos invasiva y finalmente demostró ser también una herramienta efectiva para guiar la selección de la vía de la enteroscopia (oral frente a anal) (8).

Cuando la VCE no está disponible o está contraindicada, la ESGE sugiere considerar la enteroscopia asistida por dispositivo como primera prueba diagnóstica en estos pacientes con los mismos lapsos, lo más pronto o cercano posible 
al episodio de sangrado (recomendación débil, evidencia de baja calidad). Adicionalmente, cuando se consideren lesiones ubicadas en el intestino delgado proximal, el rendimiento diagnóstico de la enteroscopia de empuje (push) es comparable con la técnica de doble balón, pero la sedación, el tiempo de examen y la exposición a rayos $\mathrm{X}$ son menores con la enteroscopia de empuje; por lo tanto, podría definirse su utilización como una herramienta de diagnóstico y tratamiento cuando se conoce que una lesión se encuentra en los segmentos más proximales del intestino delgado, lo que ahorra recursos, costos y morbilidad.

Continuando con la comparación con otros métodos diagnósticos del sangrado del intestino delgado, la VCE es superior a la angiografía mesentérica y la angioTAC para determinar la causa de sangrado. En un ensayo controlado que compara la VCE frente a la angiografía, Leung y colaboradores evaluaron el rendimiento diagnóstico y los resultados a largo plazo en 60 pacientes con sangrado manifiesto. El rendimiento diagnóstico para la VCE fue significativamente mayor que para la angiografía: $53,3 \%$ frente a 20,0 \% (diferencia de 33,3 \%; IC $95 \%: 8,9 \%-52,8 \%$ ). El riesgo acumulado de volver a sangrar en el grupo de angiografía y VCE fue de $33,3 \%$ y $16,7 \%$, respectivamente, lo que indicaba que en varios pacientes la arterografía no identificó lesiones eventualmente potenciales a ser intervenidas (9). En la actualidad se reserva la arteriografía para aquellos pacientes con sangrado del intestino delgado manifiesto hemodinámicamente inestables y, por su parte, la angioTAC multifásico para el sangrado manifiesto persistente hemodinámicamente estable sin identificación previa del origen del sangrado.

En el paciente hospitalizado con sangrado gastrointestinal oscuro manifiesto, la ESGE sugiere que la cápsula de emergencia del intestino delgado o la enteroscopia asistida por dispositivos deben considerarse durante el curso del sangrado, con la anotación de que la enteroscopia permitirá el tratamiento en el mismo procedimiento (recomendación débil, calidad moderada evidencia). La posibilidad de utilizar VCE para un sangrado manifiesto grave en curso es atractiva debido a la relativa seguridad, facilidad y viabilidad del procedimiento en esta condición. Además, ya se ha establecido que el rendimiento de la VCE temprana confiere un rendimiento diagnóstico superior (70\%), que se traduce en un mejor enfoque de manejo y en resultados positivos para el paciente. En este mismo sentido, los datos sobre el papel de la enteroscopia asistida por dispositivos de emergencia para el diagnóstico y tratamiento del sangrado manifiesto grave son limitados y a la fecha solo se presenta escasa evidencia, con series de casos pequeñas y una cantidad reducida de pacientes en quienes la EDB de emergencia se realizó dentro de las
24 horas de la presentación clínica y mostró un rendimiento diagnóstico y terapéutico del $90 \%$ (10).

¿Y en qué casos es necesario repetir una cápsula en términos de rendimiento diagnóstico? La ESGE recomienda que en casos de hemorragia manifiesta persistente o necesidad de transfusiones de sangre se justifica un enfoque alternativo. En tales pacientes, repetir la VCE puede producir un resultado positivo y especialmente en pacientes con una caída en la hemoglobina de al menos $4 \mathrm{~g} / \mathrm{dL}$ o en aquellos con un cambio en la presentación clínica de sangrado oculto a sangrado manifiesto (11). Alternativamente, la enteroscopia asistida por dispositivo o la angioTAC pueden realizarse después de una VCE inicial negativa en un paciente con sangrado persistente que puede generar un hallazgo positivo.

Finalmente, Teshima y colaboradores (11) encontraron que el rendimiento diagnóstico combinado de la EDB realizado después de una VCE previamente positiva fue del 75,0 \% (IC $95 \%: 60,1 \%-90 \%$ ). En ese mismo estudio, un análisis de subgrupos reveló que el rendimiento diagnóstico conjunto de la EDB realizada después de una VCE previamente negativa fue de $27,5 \%$ (IC $95 \%: 16,7 \%-37,8 \%$ ), que refuerza lo anteriormente mostrado en la literatura en relación con el VPN de la cápsula, pero con suficientes razones para considerar que llevar a estudios alternativos puede conseguir resultados positivos en un grupo de pacientes seleccionados.

Cinco recomendaciones sobre el sangrado del intestino delgado de las guías clínicas de la American College of Gastroenterology (ACG) (12):

1. La VCE debe considerarse como el estudio de primera línea en la investigación del intestino delgado y, por ende, del sangrado del intestino delgado.

2. Si no existe contraindicación, la cápsula siempre debería usarse antes de la enteroscopia para incrementar el rendimiento diagnóstico.

3. La enteroscopia asistida por dispositivos inicialmente se puede considerar en casos de hemorragia oscura manifiesta masiva o cuando la VCE está contraindicada o no esté disponible.

4. Si se encuentra una fuente de sangrado por la VCE o enteroscopia profunda que se asocia con anemia significativa o sangrado activo, el paciente debe ser manejado con terapia endoscópica.

5. Cuando exista sangrado agudo manifiesto en un paciente inestable debe llevarse a arteriografía urgente.

\section{CONCLUSIÓN}

El estudio del sangrado del intestino delgado actualmente cuenta con métodos diagnósticos de excelente rendi- 
miento y, aunque la VCE se considera el estudio de primera línea por las beneficios expuestos, la enteroscopia asistida por dispositivo presenta tasas de rendimiento similares con la ventaja de realizar simultáneamente el tratamiento intervencionista sobre lesiones que sangran en el intestino delgado o permite su marcación en caso de neoplasias o lesiones inflamatorias. Se dispone de otras técnicas en especial radiológicas que tienen indicaciones puntuales, principalmente cuando el estado hemodinámico del paciente no da espera a otras intervenciones, como es el caso de la arteriografía selectiva. El desarrollo continúa siendo parte de la investigación acerca de las técnicas diagnósticas, en la que se mantienen las mejores expectativas.

\section{REFERENCIAS}

1. Sandoval Riveros C, Lúquez Mindiola A, Marulanda Fernandez H, Otero Regino W. Sangrado del intestino delgado: enfoque y tratamiento. Rev Colomb Gastroenterol. 2017;32(3):245-57. https://doi.org/10.22516/25007440.156

2. Pennazio M, Spada C, Eliakim R, Keuchel M, May A, Mulder CJ, Rondonotti E, Adler SN, Albert J, Baltes P, Barbaro F, Cellier C, Charton JP, Delvaux M, Despott EJ, Domagk D, Klein A, McAlindon M, Rosa B, Rowse G, Sanders DS, Saurin JC, Sidhu R, Dumonceau JM, Hassan C, Gralnek IM. Small-bowel capsule endoscopy and deviceassisted enteroscopy for diagnosis and treatment of small bowel disorders: European Society of Gastrointestinal Endoscopy (ESGE) Clinical Guideline. Endoscopy. 2015;47(4):352-76. https://doi.org/10.1055/s-0034-1391855

3. Bonnet S, Douard R, Malamut G, Cellier C, Wind P. Intraoperative enteroscopy in the management of obscure gastrointestinal bleeding. Dig Liver Dis. 2013;45(4):277-84. https://doi.org/10.1016/j.dld.2012.07.003

4. Li Z, Liao Z, McAlindon M. Handbook of Capsule Endoscopy. Dordrecht: Springer; 2014. https://doi.org/10.1007/978-94-017-9229-5

5. Upchurch BR, Vargo JJ. Small bowel enteroscopy. Rev Gastroenterol Disord. 2008;8(3):169-77.

6. Brito HP, Ribeiro IB, Moura DTHD, Bernardo WM, Chaves DM, Kuga R, Maahs ED, Ishida RK, Moura ETHD, Moura EGHD. Video capsule endoscopy vs double-balloon enteroscopy in the diagnosis of small bowel bleeding: A systematic review and meta-analysis. World Journal of Gastrointestinal Endoscopy. 2018;10(12):400-21.
7. Lepileur L, Dray X, Antonietti M, Iwanicki-Caron I, Grigioni S, Chaput U, Di-Fiore A, Alhameedi R, Marteau P, Ducrotté P, Lecleire S. Factors associated with diagnosis of obscure gastrointestinal bleeding by video capsule enteroscopy. Clin Gastroenterol Hepatol. 2012;10(12):1376-80. https://doi.org/10.1016/j.cgh.2012.05.024

8. Li X, Chen H, Dai J, Gao Y, Ge Z. Predictive role of capsule endoscopy on the insertion route of double-balloon enteroscopy. Endoscopy. 2009;41(9):762-6. https://doi.org/10.1055/s-0029-1215009

9. Leung WK, Ho SS, Suen BY, Lai LH, Yu S, Ng EK, Ng SS, Chiu PW, Sung JJ, Chan FK, Lau JY. Capsule endoscopy or angiography in patients with acute overt obscure gastrointestinal bleeding: a prospective randomized study with long-term follow-up. Am J Gastroenterol. 2012;107(9):1370-6. https://doi.org/10.1038/ajg.2012.212

10. Mönkemüller K, Neumann H, Meyer F, Kuhn R, Malfertheiner P, Fry LC. A retrospective analysis of emergency double-balloon enteroscopy for small-bowel bleeding. Endoscopy. 2009;41(8):715-7. https://doi.org/10.1055/s-0029-1214974

11. Teshima CW, Kuipers EJ, van Zanten SV, Mensink PB. Double balloon enteroscopy and capsule endoscopy for obscure gastrointestinal bleeding: an updated meta-analysis. J Gastroenterol Hepatol. 2011;26(5):796-801. https://doi.org/10.1111/j.1440-1746.2010.06530.x

12. Gerson LB, Fidler JL, Cave DR, Leighton JA. ACG Clinical Guideline: Diagnosis and Management of Small Bowel Bleeding. Am J Gastroenterol. 2015;110(9):1265-87; quiz 1288. https://doi.org/10.1038/ajg.2015.246 